\title{
Differential Toxicity of mDia Formin-Directed Functional Agonists and Antagonists in Developing Zebrafish
}

OPEN ACCESS

Edited by:

Luc Zimmer,

Claude Bernard University Lyon 1,

France

Reviewed by:

Nicola Conran,

Universidade Estadual de Campinas,

Brazil

Maria Violetta Brundo,

Università degli Studi di Catania, Italy

*Correspondence:

Kathryn M. Eisenmann

kathryn.eisenmann@utoledo.edu

tThese authors have contributed equally to this work.

Specialty section:

This article was submitted to

Experimental Pharmacology and Drug

Discovery,

a section of the journal

Frontiers in Pharmacology

Received: 06 February 2018

Accepted: 23 March 2018

Published: 10 April 2018

Citation:

LeCorgne H, Tudosie AM, Lavik K

Su R, Becker KN, Moore S, Walia Y, Wisner A, Koehler D, Alberts AS, Williams FE and Eisenmann KM (2018) Differential Toxicity of mDia

Formin-Directed Functional Agonists and Antagonists in Developing

Zebrafish. Front. Pharmacol. 9:340 doi: 10.3389/fphar.2018.00340

\begin{abstract}
Hunter LeCorgne ${ }^{1 \dagger}$, Andrew M. Tudosie ${ }^{1 \dagger}$, Kari Lavik ${ }^{1 \dagger}$, Robin Su ${ }^{1}$, Kathryn N. Becker ${ }^{1}$, Sara Moore ${ }^{1}$, Yashna Walia ${ }^{1}$, Alexander Wisner ${ }^{2}$, Daniel Koehler ${ }^{2}$, Arthur S. Alberts ${ }^{3}$, Frederick E. Williams ${ }^{2}$ and Kathryn M. Eisenmann ${ }^{1 *}$

${ }^{1}$ Department of Cancer Biology, University of Toledo Health Science, Toledo, $\mathrm{OH}$, United States, ${ }^{2}$ Department of Pharmacology and Experimental Therapeutics, University of Toledo Health Science, Toledo, $\mathrm{OH}$, United States, ${ }^{3}$ Laboratory of Cell Structure and Signal Integration, Van Andel Research Institute, Grand Rapids, MI, United States
\end{abstract}

The mammalian Diaphanous-related (mDia) formins are cytoskeletal regulators that assemble and, in some cases, bundle filamentous actin (F-actin), as well as stabilize microtubules. The development of small molecule antagonists and agonists that interrogate $\mathrm{mDia}$ formin function has allowed us to investigate the roles of formins in disease states. A small molecule inhibitor of $\mathrm{FH} 2$ domain (SMIFH2) inhibits mDia-dependent actin dynamics and abrogates tumor cell migration and cell division in vitro and ex vivo tissue explants. mDia formin activation with small molecule intramimics IMM01/02 and mDia2-DAD peptides inhibited glioblastoma motility and invasion in vitro and ex vivo rat brain slices. However, SMIFH2, IMMs, and mDia2 DAD efficacy in vivo remains largely unexplored and potential toxicity across a range of developmental phenotypes has not been thoroughly characterized. In this study, we performed an in vivo screen of early life-stage toxicity in Danio rerio zebrafish embryos 2 days post-fertilization (dpf) in response to SMIFH2, IMM01/02, and mDia2 DAD. SMIFH2 at concentrations $\geq 5-10 \mu \mathrm{M}$ induced significant defects in developing zebrafish, including shorter body lengths, tail curvature and defective tail cellularity, craniofacial malformations, pericardial edema, absent and/or compromised vasculature function and flow, depressed heart rates and increased mortality. Conversely, IMM and mDia2 DAD peptides were minimally toxic at concentrations up to 10-20 and $50 \mu \mathrm{M}$, respectively. SMIFH2's therapeutic potential may therefore be limited by its substantial in vivo toxicity at functional concentrations. mDia formin agonism with IMMs and mDia2 DADs may therefore be a more effective and less toxic anti-invasive therapeutic approach.

Keywords: SMIFH2, intramimics, formin, zebrafish, mDia, actin cytoskeleton, invasion, xenograft

\section{INTRODUCTION}

Dynamic regulation of the cytoskeleton impacts cell motility in a variety of in vitro and in vivo tumor models. Formin family proteins regulate both actin and microtubule network dynamics. The mammalian Diaphanous (mDia) formins nucleate, elongate, and bundle filamentous actin (F-actin) (Castrillon and Wasserman, 1994; Romero et al., 2004; Kovar et al., 2006). mDia formins 
are essential to formation and function of protrusions underlying cell migration and invasion, including lamellipodia (Watanabe et al., 1997; Kurokawa and Matsuda, 2005; Eisenmann et al., 2007a; Gupton et al., 2007; Isogai et al., 2015b), filopodia (Peng et al., 2003; Yang et al., 2007; Mellor, 2010), invadopodia (Lizarraga et al., 2009), and plasma membrane blebs (Eisenmann et al., 2007a; Wyse et al., 2012). Independently, mDia formins stabilize microtubules (Gundersen and Bulinski, 1988; Tran et al., 2007; Bartolini et al., 2008; Thurston et al., 2012). Forminregulated microtubules organize tracks for vesicle transport, underlie microtentacle formation, and facilitate pro-migratory Golgi re-orientation to aid in polarized cell movement (Nobes and Hall, 1999; Ridley et al., 2003; Yamana et al., 2006; Whipple et al., 2007; Andres-Delgado et al., 2012; Morris et al., 2014; Boggs et al., 2015; Bartolini et al., 2016). Drugs like taxol have made the cytoskeleton a clinically valid target. Thus, targeting formins with small molecules may be an effective therapeutic intervention with potential to impact both the microtubule and actin dynamics during cell division and migration.

mDia-formin-directed cytoskeleton regulation is mediated through the functional Formin Homology-2 (FH2) domain (Castrillon and Wasserman, 1994; Romero et al., 2004; Kovar et al., 2006). Auto-inhibited mDia formins maintain an inactive conformation when the C-terminal Dia auto-regulatory domain (DAD) is bound to the Dia inhibitory domain (DID), blocking FH2 domain functional activities (Watanabe et al., 1999; Alberts, 2001; Higgs, 2005; Li and Higgs, 2005). Rho-GTPase binding to the GTPase-binding domain (GBD) disrupts the DID-DAD binding to enable $\mathrm{FH} 2$ functional activities, including actin filament elongation and, in some cases, bundling.

Small molecule inhibitor of FH2 (SMIFH2) is a small molecule inhibitor that blocks functional formin activity in yeast and mammalian cells (Rizvi et al., 2009). This 2thiooxodihydropyrimidine-4,6-dione derivative blocks mDia formin-mediated actin nucleation and elongation by disrupting F-actin binding. Only mDial and mDia2 $\mathrm{FH} 2$ inhibition was fully characterized biochemically in vitro for SMIFH2's effects upon rates of F-actin assembly and bundling (Rizvi et al., 2009). It remains uncertain if SMIFH2 inhibits other FH2-domain containing formin family members, as the $\mathrm{FH} 2$ domains of other non-mDia family formins (such as FMNL family members) were determined to share on average only $\sim 25 \%$ sequence identity in multiple sequence alignments (Schonichen and Geyer, 2010). Nonetheless, SMIFH2 is considered by most to be a broad antagonist against FH2 domain-containing formins. SMIFH2 also disrupts microtubule networks, and alters Golgi orientation and cell polarity (Arden et al., 2015; Isogai et al., 2015a). Formin inhibition through dominant negative constructs, RNAi knockdown and small molecule antagonism has shown to be an effective anti-invasion strategy for halting cancer cell motility in some experimental systems (Poincloux et al., 2011) and could have clinical utility, based on some in vitro cancer cell models (Arden et al., 2015; Isogai et al., 2015a; Ziske et al., 2016). SMIFH2 alters cytoskeletal protrusion formation (Rizvi et al., 2009; Wyse et al., 2012; Borinskaya et al., 2015; Fattouh et al., 2015) with mixed effects on tumor cell migration (Rizvi et al., 2009; Pettee et al., 2014; Arden et al., 2015; Isogai et al., 2015a). SMIFH2 also disrupts normal fibroblast and epithelial cell cytoskeletal networks to promote aneuploidy (Rizvi et al., 2009; Efremov et al., 2015; Kim et al., 2015).

Studies show roles for formin antagonism impeding both the microtubule, as well as actin cytoskeletal dynamics. In vitro, low to moderate SMIFH2 concentrations are welltolerated, but higher concentrations induce cytotoxic effects on mouse embryonic cells and NIH3T3 fibroblasts, HEK293T human embryonic kidney, MDA-MB-231 breast cancer, U2OS bone osteosarcoma, HCT116 colorectal carcinoma, U251 glioblastoma, and OVCA429 and ES2 ovarian carcinoma cells (Rizvi et al., 2009; Poincloux et al., 2011; Wyse et al., 2012, 2017; Pettee et al., 2014; Borinskaya et al., 2015; Fattouh et al., 2015; Isogai et al., 2015a; Ziske et al., 2016). This may be due to dual effective inhibition of both microtubule and actin dynamics within two-dimensional monolayers of cells. While $1 \mu \mathrm{M}$ SMIFH2 disrupted ovarian cancer cell monolayer viability, higher concentrations were tolerated if cells were grown in three-dimensional (3D) spheroids (Ziske et al., 2016). This finding suggests that cells organized into physiologically-relevant organoid structures may confer increased tolerance to drug toxicity and formin antagonism. Evidence describing SMIFH2 efficacy and/or toxicity in vivo, however, are limited. A role for FMNL formins was shown in developing lumenized vasculature including intersegmental vessels (ISVs) in zebrafish (Hetheridge et al., 2012; Santos-Ledo et al., 2013), and SMIFH2 suppressed lumenized ISV development (Phng et al., 2015).

Formin agonists are less well studied. mDia2 agonist intramimics, or IMMs, are small molecule agonists that activate mDia2 through disruption of the autoregulatory interaction between the DAD and DID domains (Lash et al., 2013). We and others demonstrated that IMM01 and IMM02 not only enhance F-actin assembly, yet also stabilize microtubules to a lesser degree, and in some cases, cause cell cycle arrest and suppress xenografted tumor cells from generating tumors in adult mice (Lash et al., 2013; Arden et al., 2015). We also demonstrated in glioblastoma multiforme (GBM), IMM agonists, and their pegylated- (PEG) DAD domain peptide counterparts are superior to SMIFH2-mediated antagonism in blocking directional GBM cell migration and invasion in vitro in multicellular 3D spheroids, and in an ex vivo rat brain slice model of spheroid invasion (Arden et al., 2015). Interestingly, agonists did not induce cell death in GBM cell lines, and anti-invasive effects were reversible in washout experiments. Thus, it appears that the mechanism of actin in mDia formin agonists in cells is less toxic and directed toward halting invasion, as opposed to proliferation. A more comprehensive in vivo study of efficacy and/or toxicity of mDia agonists, in particular PEG-DAD, remains untested.

Danio rerio zebrafish are highly predictive for evaluating the toxicity of soluble compounds in mammals, due in part to significant gene conservation and organ homology between zebrafish and humans/mice. Soluble compounds can be placed directly into the embryo medium for passive uptake (Goldsmith, 2004; Shin et al., 2005; McGrath and Li, 2008; Goldsmith and Jobin, 2012; Salvaggio et al., 2016). In this study, toxicity of SMIFH2, IMMs and a newly developed PEG-DAD peptide were evaluated through zebrafish embryo early life stage toxicity 
screening. We found that while IMM and PEG-DAD compounds were well tolerated in zebrafish embryos, SMIFH2 induced significant toxicity to embryos at concentrations below those shown to be functionally antagonistic to F-actin dynamics in vitro. SMIFH2's therapeutic potential may therefore be limited by substantial in vivo toxicity at functional concentrations. Our data also point toward an emerging role for mDia2 agonists as welltolerated therapeutic compounds with anti-invasive activities in several cancer types.

\section{MATERIALS AND METHODS}

\section{Zebrafish Housing, Maintenance, and Breeding}

Adult $A B$ and Tg (fli1a:EGFP) Danio rerio were from ZIRC (University of Oregon, Eugene, OR). This study was carried out in accordance with the recommendations in accordance with University of Toledo IACUC-approved regulations/protocols. Tg (fli1a:EGFP) fish express cytoplasmic EGFP within endothelial and endocardial cells, and the entire vascular system via the flila tissue-specific promoter (Lawson and Weinstein, 2002a,b). Adults were bred weekly in an Aquatic Habitats Mass Embryo Production System (MEPES) from Pentair (Minneapolis, MN). Embryos were maintained in standard embryo medium $(1 \mathrm{~L}$ filtered $\mathrm{R} / \mathrm{O}$ water with $0.6 \mathrm{~g}$ Instant Ocean Sea Salt) at $28^{\circ} \mathrm{C}$.

\section{Zebrafish Drug Toxicity Screen}

At $48 \mathrm{~h}$ post-fertilization (hpf), embryos were enzymatically dechorionated using $2 \mathrm{mg} / \mathrm{mL}$ pronase from Millipore (Burlington, MA). Healthy embryos were transferred 5/well into standard 24-well, clear, flat-bottom plates containing standard embryo medium. Time 0 images were collected at $2 \mathrm{X}$ on an EVOS-FL microscope (Olympus Plan N 2x/1.00 objective) immediately prior to treatment.

Working concentrations of $0.1-10 \mu \mathrm{M}$ SMIFH2 from Tocris (Bristol, UK) were prepared in standard embryo medium. Vehicle control was prepared by diluting equivalent DMSO (Sigma) volumes for $10 \mu \mathrm{M}$ SMIFH2 in standard embryo medium. Working concentrations of 5, 10, and $20 \mu \mathrm{M}$ IMM01 and 1, 5, and $10 \mu \mathrm{M}$ IMM02 from Thermofisher (Waltham, MA) were prepared by diluting $100 \mathrm{mM}$ stock into standard embryo medium. Vehicle controls were prepared by diluting equivalent volume of DMSO from Sigma (St Louis, MO) for $20 \mu \mathrm{M}$ IMM01 in standard embryo medium. Working concentrations of 1 , 10 , and $50 \mu \mathrm{M}$ of both PEG-DAD-M and PEG-DAD-A (DAD$M$ is the wild-type mDia2 DAD sequence with M1041, while DAD-A is M1041A substitution and is a negative control) (from New England Peptide, Gardner, MA) were prepared by diluting $10 \mathrm{mM}$ stock into standard embryo medium. Vehicle controls were prepared by diluting equivalent volume of $\mathrm{diH}_{2} \mathrm{O}$ for $50 \mu \mathrm{M}$ PEG-DADs in standard embryo medium. Embryo medium from each well was replaced with $900 \mu \mathrm{l}$ of treated embryo medium. Drug concentrations were maintained by exchanging medium every $24 \mathrm{~h}$. Treatments were in triplicate with three wells prepared for each drug condition and five embryos/well. All screens were repeated at least three independent times.

\section{Embryo Assessment and Phenotype Scoring}

After $4-144 \mathrm{~h}$ post-treatment (hpt), brightfield or fluorescent images for each embryo were acquired using an EVOS microscope with a $2 \mathrm{X}$ objective. Tricaine anesthesia was used to orient the fish in a sagittal plane in order to obtain images clearly characterizing any defects (i.e., tail curvature/degradation, edema, and head shape), and does not interfere with heart rate phenotypes (Craig et al., 2006).

Treatment mortality was based on number of surviving embryos with a visible heartbeat at each time point. Embryo heart rates in beats per minute (bpm) were determined by manually counting the embryo heart beat for $10 \mathrm{~s}$ and multiplying that value by 6 .

Early life stage toxicity defects were determined by manually examining blinded embryo images. These images were scored for tail curvature and degradation, pericardial edema, craniofacial characteristics, and development of sub-intestinal vessels (SIVs). Metamorph software measured zebrafish length from head to tail. For tail curvature, deviation observed above or below the straight edge was scored as tail curvature. Craniofacial defects (head shape) were blindly scored qualitatively as round, intermediate or square shapes with consideration of the embryo's orientation angle. SIVs are characterized as a loop of vessels protruding down into the posterior side of the embryonic sac. Fluorescence images at $21 \mathrm{hpt}$ were evaluated and SIVs were scored as either present or absent.

\section{Statistical Analysis}

Graphical representations were prepared using GraphPad Prism software. One way ANOVA analysis was used to determine statistical significance, where $p<0.05$ was considered statistically significant. Error bars represent standard errors (SE).

\section{RESULTS}

\section{SMIFH2 Induces Tail Curvature and Peripheral Cell Death in Tails of Developing Zebrafish Embryos}

We performed toxicity screens on $2 \mathrm{dpf}$ zebrafish embryos, testing 0.1-10 $\mu \mathrm{M}$ DMSO (vehicle), SMIFH2, and 1-10 $\mu \mathrm{M}$ IMMs (or DMSO) or 1-50 $\mu$ M PEG-DADs (or $\mathrm{diH}_{2} 0$ ) for up to 28 hpt. We first evaluated tail curvature and tail morphologies in treated zebrafish embryos (Figures 1A-G). The untreated, DMSO-, and $\mathrm{diH}_{2} \mathrm{O}$-treated zebrafish had straight tails at 21 hpt, as did embryos treated with all concentrations of IMM01, IMM02, and PEG-DADs, including the wild-type DAD sequence, PEG-DAD M, as well as the alanine-substituted negative control, PEG-DAD A (Figures 1F,G). Embryos treated with 0.1 and $1 \mu \mathrm{M}$ SMIFH2 presented with straight tails (Figures 1A,E). However, $10 \mu \mathrm{M}$ SMIFH2 induced dramatic and statistically significant upwards tail curvature $21 \mathrm{hpt}$ ( $>5$-fold increase relative to controls), and curvatures appeared to begin posterior of the end of the yolk extension (Figures 1A,E). Furthermore, the upwardly curved tails from $10 \mu \mathrm{M}$ SMIFH2-treated embryos 

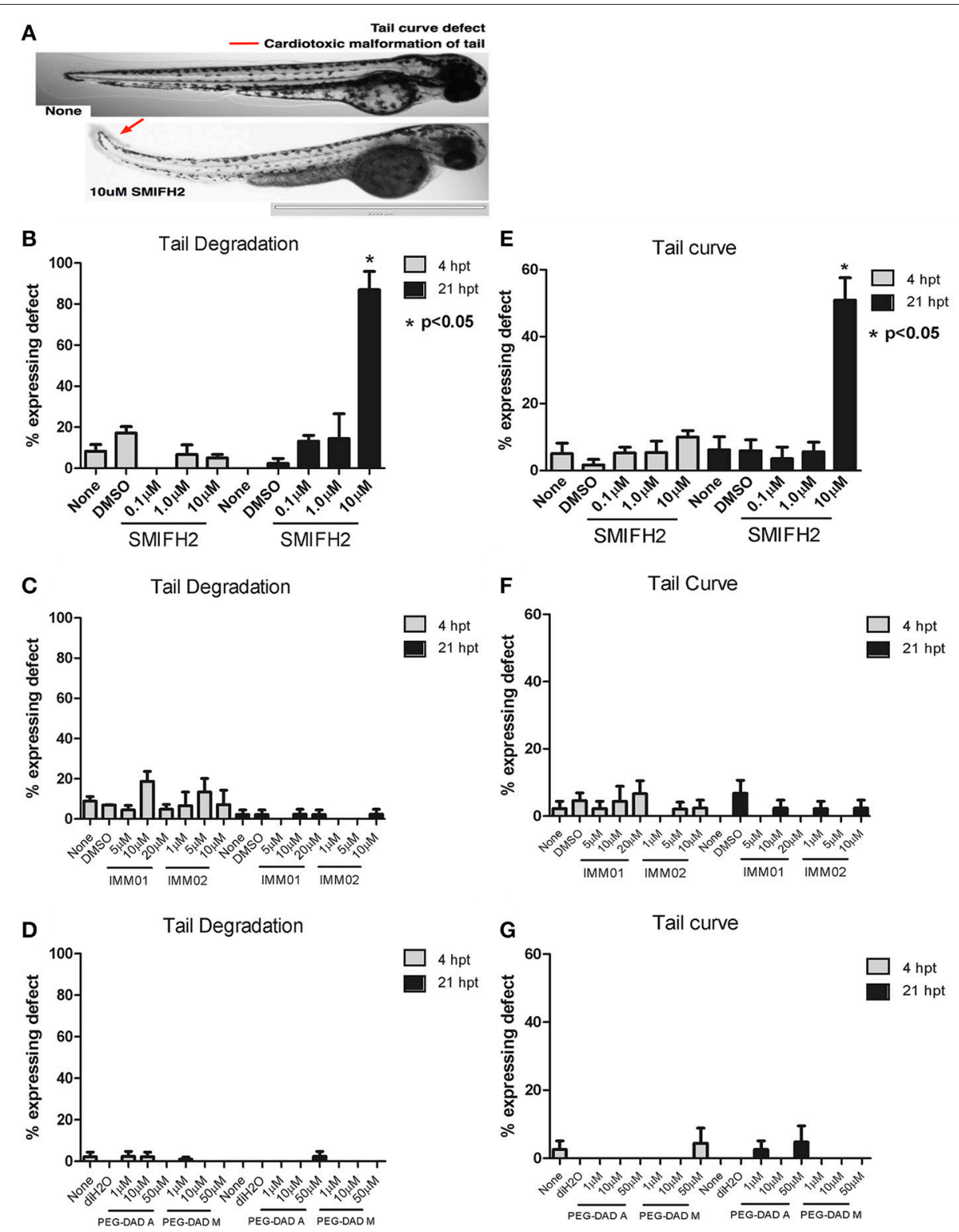

FIGURE 1 | Formin antagonism induces tail curving and peripheral tail cell death in developing zebrafish embryos. (A) Representative 2x images at 21 hpt untreated (None) and in $10 \mu \mathrm{M}$ SMIFH2 (arrow indicates peripheral tail cell death). Scale bar $=2,000 \mu \mathrm{m}$. (B-D) Tail degradation was scored for embryos in each untreated, vehicle, and SMIFH2 (B), IMM (C), and PEG-DAD (D) drug conditions. (E-G). Tail curvature was scored for embryos in each untreated, vehicle, and SMIFH2 (E), IMM (F), and PEG-DAD (G) drug condition. The screens were performed in triplicate, five embryos/well and the experiment was repeated three times. $p$-Value is relative to untreated condition. Error bars indicate SE.

also showed highly disorganized peripheral tail cellular structure relative to controls (Figures $\mathbf{1 B}-\mathbf{D}$ ), accompanied by tail tip malformation with discoloration and deterioration, indicative of peripheral tail cell death. At $>10 \mu \mathrm{M}$ SMIFH2, cells rapidly sloughed off tails within $21 \mathrm{hpt}$ (not shown). This may be indicative of a lack of perfusion caused by overall cardiotoxicity of SMIFH2 and mDia functional suppression. In contrast, IMM and PEG-DAD mDia2 agonist-treated embryos did not have compromised tail structures, relative to untreated and vehicle treated controls.

\section{Antagonism Induces Craniofacial Malformations in Developing Zebrafish Embryos}

We next assessed craniofacial mutations observed in treated zebrafish embryos. Untreated, DMSO-, and $\mathrm{diH}_{2} \mathrm{O}$-treated embryos had characteristic rounded facial structures, elongated snouts, with two distinct eyes (Figure 2A, upper panel), as did embryos treated with all concentrations of IMM01, IMM02, PEG-DAD-M, and PEG-DAD-A (Figures 2A,C,D). Craniofacial features in embryos treated with 0.1 and $1 \mu \mathrm{M}$ SMIFH2 
A

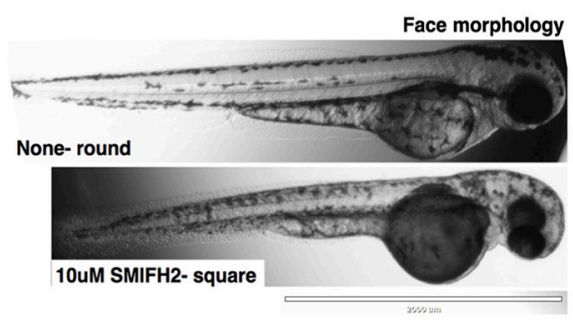

C

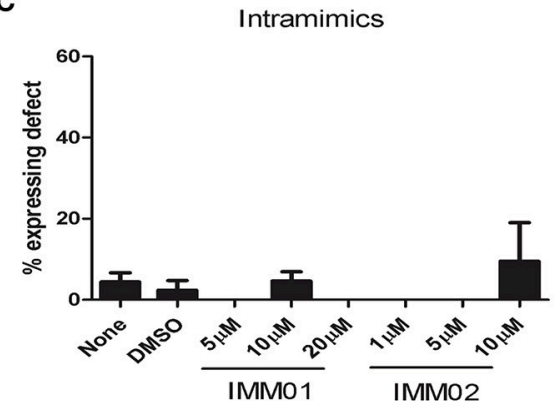

B

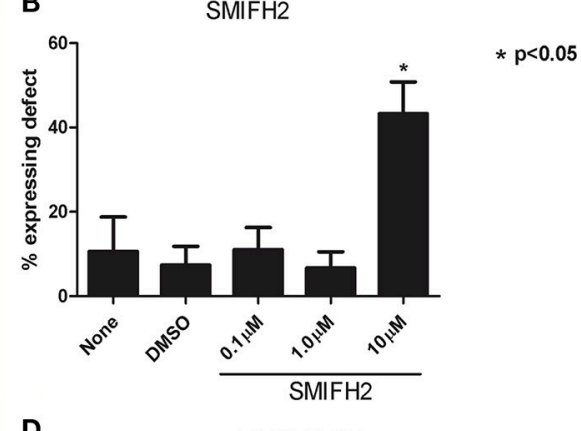

D

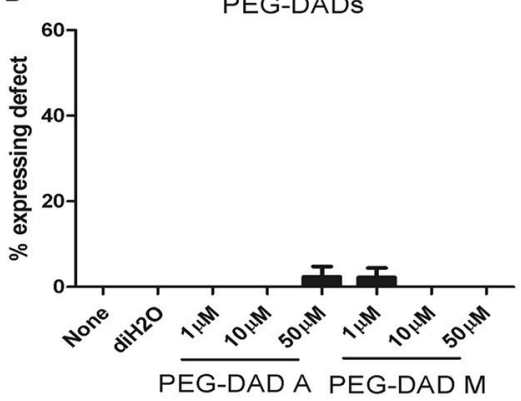

FIGURE 2 | Formin antagonism induces craniofacial defects in developing zebrafish embryos. (A) Representative 2x images at 21 hpt untreated (None) and in $10 \mu \mathrm{M}$ SMIFH2. (B-D) Craniofacial defects were scored for embryos in each untreated, vehicle, and SMIFH2 (B), IMM (C), and PEG-DAD (D) drug conditions. The screens were performed in triplicate, five embryos/well and the experiment was repeated three times. Values are represented as \% expressing squared faces per condition. $p$ value is relative to untreated condition. Error bars indicate SE. Scale bars $=2,000 \mu \mathrm{m}$.

were unaffected, while $10 \mu \mathrm{M}$ SMIFH2-treated embryos had a statistically significant increase in blunted, shortened, or squared-off facial morphologies (5-fold increase relative to controls; Figures 2A,B), indicative of a developing jaw defect and perhaps due to deficiencies in local vascularization or circulation impacting jaw growth (Teraoka et al., 2002). The eyes were also misplaced, fused, or near-fused in SMIFH2-treated embryos having the squared craniofacial morphology.

\section{Antagonism Induces Pericardial Edema in Developing Zebrafish Embryos}

We next quantified pericardial edema in treated embryos. In control, DMSO-, and $\mathrm{diH}_{2} \mathrm{O}$-treated embryos there was no evidence of pericardial edema (Figure 3). Similarly, there was no edema in those embryos treated with increasing concentrations of IMM01, IMM02, PEG-DAD-M, and PEG-DAD-A, relative to controls (Figures 3C,D). Embryos treated with 0.1 and $1 \mu \mathrm{M}$ SMIFH2 were also normal with respect to pericardial edema; However, in $10 \mu \mathrm{M}$ SMIFH2-treated embryos, we observed clear masses anterior to the heart yet posterior to the head (Figure 3A). Nearly $90 \%$ of $10 \mu \mathrm{M}$ SMIFH2-treated embryos had pericardial edema, relative to $10 \%$ of embryos treated with respective vehicle or mDia2 agonists (Figure 3B). Pericardial edema has previously been attributed to increases in vascular permeability of the proximal vessels, or impaired development of the heart itself (Hill et al., 2004).

\section{Antagonism Impairs Vascular Flow in Developing Zebrafish Embryos}

We next wished to investigate the dynamics of vascular flow within treated embryos upon mDia antagonism. Hence, we treated developing zebrafish embryos with DMSO, or $0.1-10 \mu \mathrm{M}$ SMIFH2 for $4 \mathrm{hpt}$. Live imaging of the cardinal vein anterior to the urogenital opening of tricaine-treated embryos was then performed to assess vascular flow posterior to the yolk extension. The vascular flow rates of 0.1 and $1 \mu \mathrm{M}$ SMIFH2-treated embryos resembled untreated or DMSO-treated embryos (Movies S1-S4, respectively). A striking difference was seen in 5 and $10 \mu \mathrm{M}$ SMIFH2-treated embryos, where vascular flow was suppressed and nearly halted, respectively (Movies S5, S6).

\section{Antagonism Impacts Heart Rates in Developing Zebrafish Embryos}

Finally, we assessed heart rates in developing embryos treated with IMM01, IMM02, PEG-DADs, and SMIFH2 through $21 \mathrm{hpt.}$ Heart rates for untreated, DMSO-, $\mathrm{diH}_{2} \mathrm{O}-$, IMM01-, IMM02-, PEG-DAD-A-, and PEG-DAD-M- and 0.1 $\mu \mathrm{M}$ SMIFH2-treated embryos remained near 120-130 bpm, with little variation across $21 \mathrm{hpt}$ (Figure 4). Exposure to $1 \mu \mathrm{M}$ SMIFH2 suppressed bpm about $10 \%$ relative to controls, and this was nearly recovered to control levels within 21 hpt (Figure 4A). Embryos treated with $10 \mu \mathrm{M}$ SMIFH2 revealed a rapid heart rate decline as early as 4 hpt. We observed a rapid decline in survival by $21 \mathrm{hpt}$ in these embryos. 

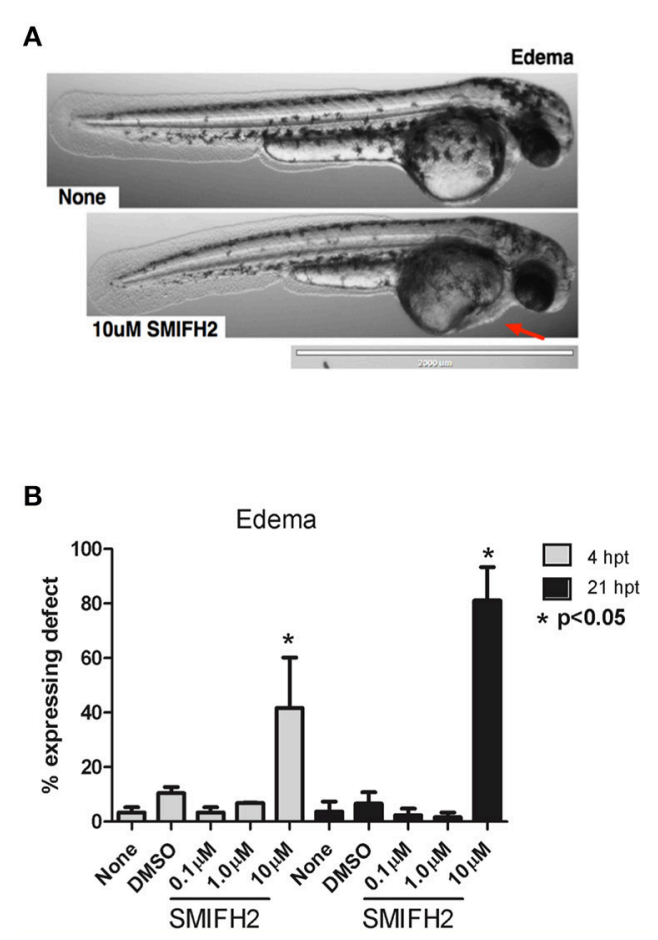

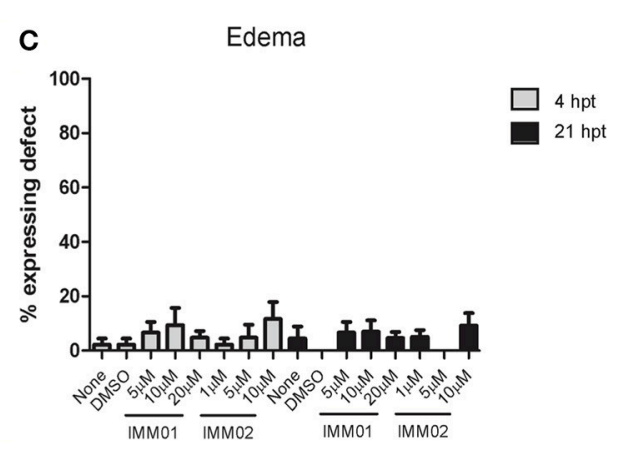

D

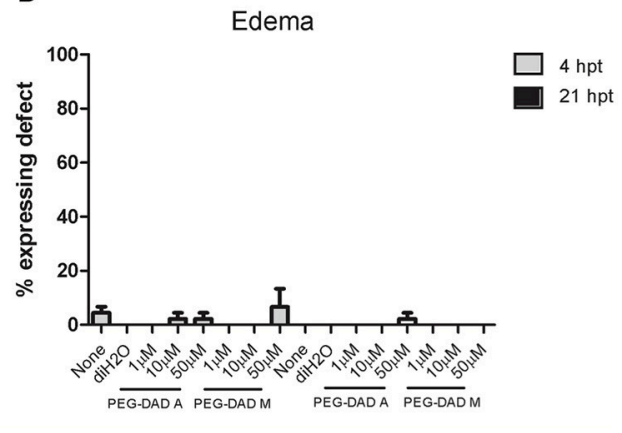

FIGURE 3 | Formin antagonism induces pericardial edema in developing zebrafish embryos. (A) Representative $2 x$ images at 21 hpt without treatment (None) and in $10 \mu$ M SMIFH2 (arrow indicates edema). Scale bar = 2,000 $\mu \mathrm{m}$. (B-D) Pericardial edema was scored for embryos in each untreated, vehicle, and SMIFH2 (B), IMM (C), and PEG-DAD (D) drug conditions. The screens were performed in triplicate, five embryos/well and the experiment was repeated three times. $p$-value is relative to untreated condition. Error bars indicate SE.

\section{DISCUSSION}

As major regulators of cellular functions including cytokinesis, vesicular trafficking, and cell motility, mDia formins were proposed as therapeutic targets in a wide-variety of pathologies, including invasive and metastatic cancers. There are at least two approaches to manipulating formin activity: antagonism and agonism. Both have been reported to target both the actin and microtubule cytoskeletons to varying degrees in different two-dimensional cellular models. An antagonist, the small molecule inhibitor SMIFH2, is a widely-used tool used to study mDia function in vitro in a variety of human cells. This study reveals cytotoxicity in vivo in developing zebrafish at $\geq 5-10 \mu \mathrm{M}$ SMIFH2. Alternatively, mDia2 intramimics, IMM01 and IMM02, as well as PEG-DADs induced no significant cytotoxicity. We documented defects in embryo lengths, embryo tail curves and cellularity, pericardial edema, craniofacial development, vascular development, and heart rates. Ultimately zebrafish embryos treated with $\geq 10 \mu \mathrm{M}$ SMIFH2 were no longer viable.

Although this study was performed in developing zebrafish embryos, embryo toxicity screens are highly predictive of mammalian drug toxicity (Langheinrich, 2003; Zhang et al., 2003; Kari et al., 2007). Zebrafish are anatomically, physiologically and molecularly similar to mammals and most major organs, including nervous system, cardiovascular system, intestines, liver, and kidneys are established by $5 \mathrm{dpf}$ (McGrath and Li, 2008). The circulatory system of zebrafish is a common site of drug toxicity. Zebrafish embryos are ideal for assessing cardiotoxicity as the heart is formed and functional by $26 \mathrm{hpf}$ (Baker et al., 1997; Chakravarthy et al., 2014), and all major vessels are present with active circulation by $28 \mathrm{hpf}$ (Kari et al., 2007). At $28 \mathrm{hpf}$, angiogenesis is underway within the anterior somites, consistent with tail curvature and cellular defects in SMIFH2treated embryos (Figure 2). Zebrafish embryos survive for up to a week without intact circulation (Stainier, 2001; Kari et al., 2007), supporting our findings that embryos persist despite lack of blood circulation while developing pericardial edema and cranial-facial abnormalities.

SMIFH2's toxicity at $\geq 5-10 \mu \mathrm{M}$ in developing zebrafish embryos may caution against its therapeutic utility in mDia formin antagonism. SMIFH2 concentrations of $\geq 10 \mu \mathrm{M}$ predominate in vitro tumor cell invasion studies (Arden et al., 2015; Isogai et al., 2015a; Ziske et al., 2016; Wyse et al., 2017). However, purified biochemical assays (Rizvi et al., 2009) indicated that $4 \mu \mathrm{M}$ SMIFH2 blocked mDia formin-dependent actin nucleation and elongation, whereas $2.5 \mu \mathrm{M}$ induced actin cable de-polymerization. Thus, at lower concentrations, SMIFH2 is effective- at least in a closed, purified system. In vitro in normal cells, $2.5 \mu \mathrm{M}$ SMIFH2 disrupted cytokinesis in NIH3T3 fibroblasts and $5 \mu \mathrm{M}$ SMIFH2 dramatically decreased MEF spreading (Iskratsch et al., 2013). SMIFH2 (0.1-1 $\mu \mathrm{M})$ 


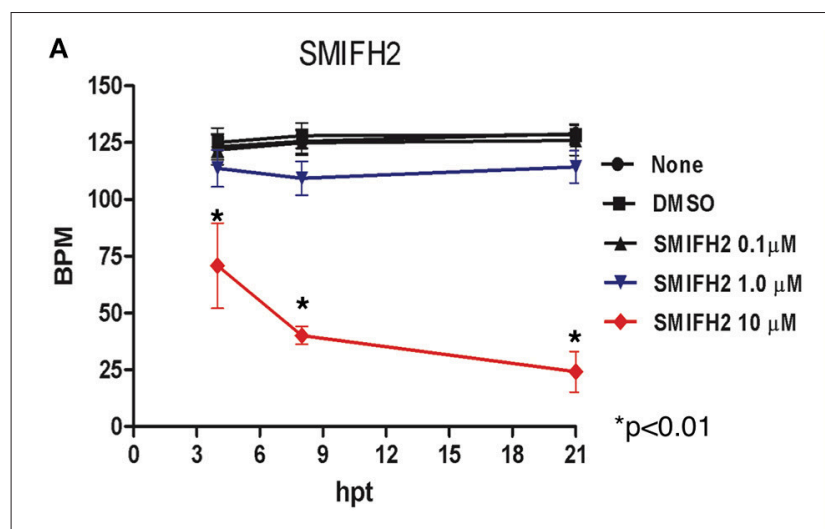

B
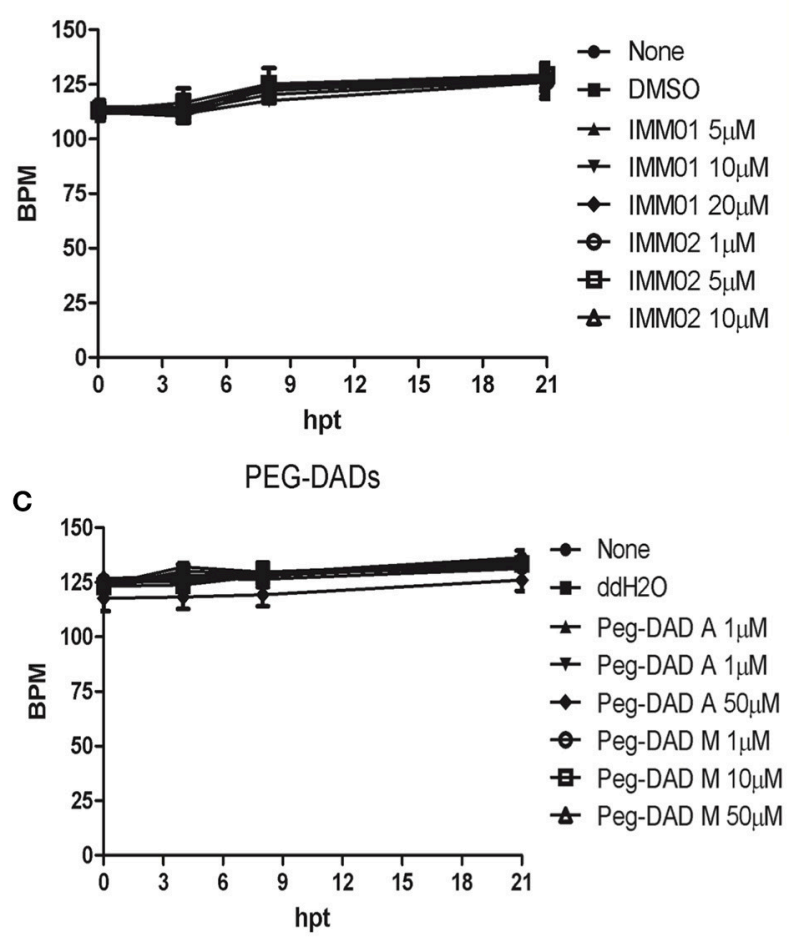

FIGURE 4 | Formin antagonism induces heart rate depression in developing zebrafish embryos. Heart rates were scored in: untreated, vehicle, SMIFH2 (A), IMM01 and IMM02 (B), and PEG-DAD-A and PEG-DAD-M (C). The screens were performed in triplicate, five embryos/well and the experiment was repeated three times. Error bars represent SE.

also enhanced Taxol or Cisplatin anti-proliferative effects in ovarian cancer spheroid cultures (Ziske et al., 2016). Here, no significant early-life stage embryo toxicity was detected at 0.1 and $1 \mu \mathrm{M}$ SMIFH2, but $5 \mu \mathrm{M}$ caused substantial cardiotoxic defects in embryos (data not shown) and impaired vascular flow (Movie S5) through 21 and $4 \mathrm{hpt}$, respectively. Recently it was suggested that $5 \mu \mathrm{M}$ SMIFH2 treatment for $15 \mathrm{~h}$ starting at $31 \mathrm{hpf}$ in zebrafish embryos suppressed F-actin stability at endothelial junctions, as serrated F-actin cables were increased (an indicator of F-actin remodeling) (Phng et al., 2015). Further studies are warranted to definitively assess the functional efficacy of SMIFH2-directed mDia formin inhibition at $\leq 5 \mu \mathrm{M}$ both in vitro and in vivo and to ascertain if both the microtubule and actin filament systems are being impacted. Cell-type specificity in toxicity may also exist in vitro and in vivo, as we previously showed 10 and $20 \mu \mathrm{M}$ SMIFH2 minimally impacted ES-2 and SKOV3 ovarian cancer 3D multi-cellular spheroid viability (Ziske et al., 2016), while $40 \mu \mathrm{M}$ SMIFH2 was not acutely toxic in treated adult rat brain slices cultured up to $48 \mathrm{~h}$ (Arden et al., 2015). SMIFH2 toxicity studies in different zebrafish developmental stages (i.e., juvenile, adult) are also currently in progress.

Our results support previous studies conducted in mouse models suggesting that formin antagonism can have a significant effect on homeostatic systems. DRF1 (encoding mDial protein) knockout mice exhibited disrupted myelopoiesis and erythropoiesis resembling the pre-leukemic human diseases chronic myeloproliferative syndrome (MPS) and myelodysplastic syndrome (MDS) (Peng et al., 2007). mDial knockout mice also showed signs of dehydration, anemia, and hepatic dysplasia (Peng et al., 2007), and had reduced numbers of T lymphocytes in the bone marrow, spleen, thymus, and blood (Eisenmann et al., 2007b). Furthermore, DRF2 (encoding mDia3 protein) suppression was correlated with primary ovarian failure (Wynshaw-Boris et al., 1997; Bione et al., 1998; Tominaga et al., 2002), while in DRF3 (encoding mDia2 protein) knockdown cells and mice, severe defects in erythrocyte maturation and enucleation were seen (Ji et al., 2008; Watanabe et al., 2013; Mei et al., 2016). These data suggest a critical role for mDia formins during both hematopoiesis and hemostasis, thus indicating caution in inhibiting global or, in some cases, tissue-specific $\mathrm{mDia}$ expression and/or functional antagonism in vivo in clinical applications.

In contrast, formin activation and not functional suppression may be more appropriate clinical strategy to target tumor cell motility, and potentially proliferation. Whereas, mDia2 suppression drove alternative modes of tumor cell motility in cervical, ovarian, breast, and prostate cancers, and hepatocarcinoma models (Eisenmann et al., 2007a; Di Vizio et al., 2009; Hager et al., 2012; Wyse et al., 2012; Pettee et al., 2014), glioblastoma cells in vitro and ex vivo spheroid-rat brain slice models showed IMM-induced mDia2 agonism more effectively blocked glioblastoma migration and invasion than antagonism, with no toxicity in rat brain slice explants (Arden et al., 2015). IMM compounds decreased hepatocarcinoma tumor burden in vivo in mice with limited side effects (Lash et al., 2013). Thus, endogenous mDia formin activation may ultimately prove a more viable therapeutic avenue to targeting tumor cell motility in future studies.

\section{AUTHOR CONTRIBUTIONS}

HL, AT, KL, RS, KB, SM, YW, AW, and DK: performed the experiments; HL, AT, and KL: drafted the manuscript; KE, AA, and FW: conceived of the experiments; KE and FW: provided funding sources and edited the paper; AA: provided invaluable reagents. All authors read and approved the submitted version (except AA, who passed away prior to submission). 


\section{ACKNOWLEDGMENTS}

The authors thank the University of Toledo Department of Laboratory and Animal Research staff and supervisors and Drs. William Maltese and Andrea Nestor-Kalinoski and Mr. Peterson Schwifty for helpful discussions. We thank Ms. Krista Pettee for help with statistical analyses. We thank the late, great AA for years of creative, solid scientific input in understanding formins, and the many off-site lab meetings at the local pub or

\section{REFERENCES}

Alberts, A. S. (2001). Identification of a carboxyl-terminal diaphanous-related formin homology protein autoregulatory domain. J. Biol. Chem. 276, 2824-2830. doi: 10.1074/jbc.M006205200

Andrés-Delgado, L., Antón, O. M., Bartolini, F., Ruiz-Sáenz, A., Correas, I., Gundersen, G. G., et al. (2012). INF2 promotes the formation of detyrosinated microtubules necessary for centrosome reorientation in T cells. J. Cell Biol. 198, 1025-1037. doi: 10.1083/jcb.201202137

Arden, J. D., Lavik, K. I., Rubinic, K. A., Chiaia, N., Khuder, S. A., Howard, M. J., et al. (2015). Small-molecule agonists of mammalian Diaphanous-related (mDia) formins reveal an effective glioblastoma anti-invasion strategy. Mol. Biol. Cell 26, 3704-3718. doi: 10.1091/mbc.E14-11-1502

Baker, K., Warren, K. S., Yellen, G., and Fishman, M. C. (1997). Defective "pacemaker" current (Ih) in a zebrafish mutant with a slow heart rate. Proc. Natl. Acad. Sci. U.S.A. 94, 4554-4559. doi: 10.1073/pnas.94.9.4554

Bartolini, F., Andres-Delgado, L., Qu, X., Nik, S., Ramalingam, N., Kremer, L., et al. (2016). An mDia1-INF2 formin activation cascade facilitated by IQGAP1 regulates stable microtubules in migrating cells. Mol. Biol. Cell 27, 1797-1808. doi: 10.1091/mbc.E15-07-0489

Bartolini, F., Moseley, J. B., Schmoranzer, J., Cassimeris, L., Goode, B. L., and Gundersen, G. G. (2008). The formin mDia2 stabilizes microtubules independently of its actin nucleation activity. J. Cell Biol. 181, 523-536. doi: $10.1083 /$ jcb.200709029

Bione, S., Sala, C., Manzini, C., Arrigo, G., Zuffardi, O., Banfi, S., et al. (1998). A human homologue of the Drosophila melanogaster diaphanous gene is disrupted in a patient with premature ovarian failure: evidence for conserved function in oogenesis and implications for human sterility. Am. J. Hum. Genet. 62, 533-541. doi: 10.1086/301761

Boggs, A. E., Vitolo, M. I., Whipple, R. A., Charpentier, M. S., Goloubeva, O. G., Ioffe, O. B., et al. (2015). $\alpha$-Tubulin acetylation elevated in metastatic and basal-like breast cancer cells promotes microtentacle formation, adhesion, and invasive migration. Cancer Res. 75, 203-215. doi: 10.1158/0008-5472.CAN-13-3563

Borinskaya, S., Velle, K. B., Campellone, K. G., Talman, A., Alvarez, D., Agaisse, H., et al. (2015). Integration of linear and dendritic actin nucleation in Nck-induced actin comets. Mol. Biol. Cell 27, 247-259. doi: 10.1091/mbc.E14-11-1555

Castrillon, D. H., and Wasserman, S. A. (1994). diaphanous is required for cytokinesis in Drosophila and shares domains of similarity with the products of the limb deformity gene. Development 120,3367-3377.

Chakravarthy, S., Sadagopan, S., Nair, A., and Sukumaran, S. K. (2014). Zebrafish as an in vivo high-throughput model for genotoxicity. Zebrafish 11, 154-166. doi: $10.1089 /$ zeb.2013.0924

Craig, M. P., Gilday, S. D., and Hove, J. R. (2006). Dose-dependent effects of chemical immobilization on the heart rate of embryonic zebrafish. Lab Anim. 35, 41-47. doi: 10.1038/laban1006-41

Di Vizio, D., Kim, J., Hager, M. H., Morello, M., Yang, W., Lafargue, C. J., et al. (2009). Oncosome formation in prostate cancer: association with a region of frequent chromosomal deletion in metastatic disease. Cancer Res. 69, 5601-5609. doi: 10.1158/0008-5472.CAN-08-3860

Efremov, Y. M., Dokrunova, A. A., Efremenko, A. V., Kirpichnikov, M. P., Shaitan, K. V., and Sokolova, O. S. (2015). Distinct impact of targeted actin cytoskeleton reorganization on mechanical properties of normal mountain bike trail. This work was supported by the University of Toledo Interdisciplinary Research Initiation Award (KE and FW), R01CA151632 (KE), and the Toledo Foundation (KE).

\section{SUPPLEMENTARY MATERIAL}

The Supplementary Material for this article can be found online at: https://www.frontiersin.org/articles/10.3389/fphar. 2018.00340/full\#supplementary-material and malignant cells. Biochim. Biophys. Acta 1853(11 Pt B), 3117-3125. doi: 10.1016/j.bbamcr.2015.05.008

Eisenmann, K. M., Harris, E. S., Kitchen, S. M., Holman, H. A., Higgs, H. N., and Alberts, A. S. (2007a). Dia-Interacting protein modulates forminmediated actin assembly at the cell cortex. Curr. Biol. 17, 579-591. doi: 10.1016/j.cub.2007.03.024

Eisenmann, K. M., West, R. A., Hildebrand, D., Kitchen, S. M., Peng, J., Sigler, R., et al. (2007b). T cell responses in mammalian diaphanousrelated formin mDial knock-out mice. J. Biol. Chem. 282, 25152-25158. doi: 10.1074/jbc.M703243200

Fattouh, R., Kwon, H., Czuczman, M. A., Copeland, J. W., Pelletier, L., Quinlan, M. E., et al. (2015). The diaphanous-related formins promote protrusion formation and cell-to-cell spread of Listeria monocytogenes. J. Infect. Dis. 211, 1185-1195. doi: 10.1093/infdis/jiu546

Goldsmith, J. R., and Jobin, C. (2012). Think small: zebrafish as a model system of human pathology. J. Biomed. Biotechnol. 2012:817341. doi: $10.1155 / 2012 / 817341$

Goldsmith, P. (2004). Zebrafish as a pharmacological tool: the how, why and when. Curr. Opin. Pharmacol. 4, 504-512. doi: 10.1016/j.coph.2004.04.005

Gundersen, G. G., and Bulinski, J. C. (1988). Selective stabilization of microtubules oriented toward the direction of cell migration. Proc. Natl. Acad. Sci. U.S.A. 85, 5946-5950. doi: 10.1073/pnas.85.16.5946

Gupton, S. L., Eisenmann, K., Alberts, A. S., and Waterman-Storer, C. M. (2007). mDia2 regulates actin and focal adhesion dynamics and organization in the lamella for efficient epithelial cell migration. J. Cell Sci. 120, 3475-3487. doi: $10.1242 /$ jcs.006049

Hager, M. H., Morley, S., Bielenberg, D. R., Gao, S., Morello, M., Holcomb, I. N., et al. (2012). DIAPH3 governs the cellular transition to the amoeboid tumour phenotype. EMBO Mol. Med. 4, 743-760. doi: 10.1002/emmm.201200242

Hetheridge, C., Scott, A. N., Swain, R. K., Copeland, J. W., Higgs, H. N., Bicknell, R., et al. (2012). The formin FMNL3 is a cytoskeletal regulator of angiogenesis. J. Cell Sci. 125(Pt 6), 1420-1428. doi: 10.1242/jcs.091066

Higgs, H. N. (2005). Formin proteins: a domain-based approach. Trends Biochem. Sci. 30, 342-353. doi: 10.1016/j.tibs.2005.04.014

Hill, A. J., Bello, S. M., Prasch, A. L., Peterson, R. E., and Heideman, W. (2004). Water permeability and TCDD-induced edema in zebrafish early-life stages. Toxicol. Sci. 78, 78-87. doi: 10.1093/toxsci/kfh056

Iskratsch, T., Yu, C. H., Mathur, A., Liu, S., Stevenin, V., Dwyer, J., et al. (2013). FHOD1 is needed for directed forces and adhesion maturation during cell spreading and migration. Dev. Cell 27, 545-559. doi: 10.1016/j.devcel.2013.11.003

Isogai, T., van der Kammen, R., and Innocenti, M. (2015a). SMIFH2 has effects on formins and p53 that perturb the cell cytoskeleton. Sci. Rep. 5:9802. doi: 10.1038/srep09802

Isogai, T., Van der Kammen, R., Leyton-Puig, D., Kedziora, K. M., Jalink, K., and Innocenti, M. (2015b). Initiation of lamellipodia and ruffles involves cooperation between mDial and the Arp2/3 complex. J. Cell Sci. 128, 3796-3810. doi: $10.1242 /$ jcs. 176768

Ji, P., Jayapal, S. R., and Lodish, H. F. (2008). Enucleation of cultured mouse fetal erythroblasts requires Rac GTPases and mDia2. Nat. Cell Biol. 10, 314-321. doi: $10.1038 /$ ncb1693

Kari, G., Rodeck, U., and Dicker, A. P. (2007). Zebrafish: an emerging model system for human disease and drug discovery. Clin. Pharmacol. Ther. 82, 70-80. doi: 10.1038/sj.clpt.6100223 
Kim, H. C., Jo, Y. J., Kim, N. H., and Namgoong, S. (2015). Small molecule inhibitor of formin homology 2 domains (SMIFH2) reveals the roles of the formin family of proteins in spindle assembly and asymmetric division in mouse oocytes. PLoS ONE 10:e0123438. doi: 10.1371/journal.pone.0123438

Kovar, D. R., Harris, E. S., Mahaffy, R., Higgs, H. N., and Pollard, T. D. (2006). Control of the assembly of ATP- and ADP-actin by formins and profilin. Cell 124, 423-435. doi: 10.1016/j.cell.2005.11.038

Kurokawa, K., and Matsuda, M. (2005). Localized RhoA activation as a Requirement for the induction of membrane ruffling. Mol. Biol. Cell 16, 4294-4303. doi: 10.1091/mbc.E04-12-1076

Langheinrich, U. (2003). Zebrafish: a new model on the pharmaceutical catwalk. Bioessays 25, 904-912. doi: 10.1002/bies.10326

Lash, L. L., Wallar, B. J., Turner, J. D., Vroegop, S. M., Kilkuskie, R. E., Kitchen-Goosen, S. M., et al. (2013). Small-molecule intramimics of formin autoinhibition: a new strategy to target the cytoskeletal remodeling machinery in cancer cells. Cancer Res. 73, 6793-6803. doi: 10.1158/0008-5472.CAN-13-1593

Lawson, N. D., and Weinstein, B. M. (2002a). Arteries and veins: making a difference with zebrafish. Nat. Rev. Genet. 3, 674-682. doi: 10.1038/nrg888

Lawson, N. D., and Weinstein, B. M. (2002b). In vivo imaging of embryonic vascular development using transgenic zebrafish. Dev. Biol. 248, 307-318. doi: 10.1006/dbio.2002.0711

Li, F., and Higgs, H. N. (2005). Dissecting requirements for auto-inhibition of actin nucleation by the formin, mDial. J. Biol. Chem. 280, 6986-6992. doi: $10.1074 /$ jbc.M411605200

Lizarraga, F., Poincloux, R., Romao, M., Montagnac, G., Le Dez, G., Bonne, I., et al. (2009). Diaphanous-related formins are required for invadopodia formation and invasion of breast tumor cells. Cancer Res. 69, 2792-2800. doi: 10.1158/0008-5472.CAN-08-3709

McGrath, P., and Li, C. Q. (2008). Zebrafish: a predictive model for assessing drug-induced toxicity. Drug Discov. Today 13, 394-401. doi: 10.1016/j.drudis.2008.03.002

Mei, Y., Zhao, B., Yang, J., Gao, J., Wickrema, A., Wang, D., et al. (2016). Ineffective erythropoiesis caused by binucleated late-stage erythroblasts in mDia2 hematopoietic specific knockout mice. Haematologica 101:e1-5. doi: 10.3324/haematol.2015.134221

Mellor, H. (2010). The role of formins in filopodia formation. Biochim. Biophys. Acta 1803, 191-200. doi: 10.1016/j.bbamcr.2008.12.018

Morris, E. J., Nader, G. P., Ramalingam, N., Bartolini, F., and Gundersen, G. G. (2014). Kif4 interacts with EB1 and stabilizes microtubules downstream of Rho-mDia in migrating fibroblasts. PLoS ONE 9:e91568. doi: 10.1371/journal.pone.0091568

Nobes, C. D., and Hall, A. (1999). Rho GTPases control polarity, protrusion, and adhesion during cell movement. J. Cell Biol. 144, 1235-1244.

Peng, J., Kitchen, S. M., West, R. A., Sigler, R., Eisenmann, K. M., and Alberts, A. S. (2007). Myeloproliferative defects following targeting of the Drf1 gene encoding the mammalian diaphanous related formin mDial. Cancer Res. 67, 7565-7571. doi: 10.1158/0008-5472.CAN-07-1467

Peng, J., Wallar, B. J., Flanders, A., Swiatek, P. J., and Alberts, A. S. (2003). Disruption of the Diaphanous-related formin Drf1 gene encoding mDial reveals a role for Drf3 as an effector for Cdc42. Curr. Biol. 13, 534-545. doi: 10.1016/S0960-9822(03)00170-2

Pettee, K. M., Dvorak, K. M., Nestor-Kalinoski, A. L., and Eisenmann, K. M. (2014). An mDia2/ROCK signaling axis regulates invasive egress from epithelial ovarian cancer spheroids. PLoS ONE 9:e90371. doi: 10.1371/journal.pone.0090371

Phng, L. K., Gebala, V., Bentley, K., Philippides, A., Wacker, A., Mathivet, T., et al. (2015). Formin-mediated actin polymerization at endothelial junctions is required for vessel lumen formation and stabilization. Dev. Cell 32, 123-132. doi: 10.1016/j.devcel.2014.11.017

Poincloux, R., Collin, O., Lizarraga, F., Romao, M., Debray, M., Piel, M., et al. (2011). Contractility of the cell rear drives invasion of breast tumor cells in 3D Matrigel. Proc. Natl. Acad. Sci. U.S.A. 108, 1943-1948. doi: $10.1073 /$ pnas. 1010396108

Ridley, A. J., Schwartz, M. A., Burridge, K., Firtel, R. A., Ginsberg, M. H., Borisy, G. G., et al. (2003). Cell migration: integrating signals from front to back. Science 302, 1704-1709. doi: 10.1126/science.1092053
Rizvi, S. A., Neidt, E. M., Cui, J., Feiger, Z., Skau, C. T., Gardel, M. L., et al. (2009). Identification and characterization of a small molecule inhibitor of formin-mediated actin assembly. Chem. Biol. 16, 1158-1168. doi: $10.1016 /$ j.chembiol.2009.10.006

Romero, S., Le Clainche, C., Didry, D., Egile, C., Pantaloni, D., and Carlier, M. F. (2004). Formin is a processive motor that requires profilin to accelerate actin assembly and associated ATP hydrolysis. Cell 119, 419-429. doi: 10.1016/j.cell.2004.09.039

Salvaggio, A., Marino, F., Albano, M., Pecoraro, R., Camiolo, G., Tibullo, D., et al. (2016). Toxic effects of zinc chloride on the bone development in danio rerio (Hamilton, 1822). Front. Physiol. 7:153. doi: 10.3389/fphys.2016.00153

Santos-Ledo, A., Jenny, A., and Marlow, F. L. (2013). Comparative gene expression analysis of the fmnl family of formins during zebrafish development and implications for tissue specific functions. Gene Expr. Patterns 13, 30-37. doi: 10.1016/j.gep.2012.09.002

Schönichen, A., and Geyer, M. (2010). Fifteen formins for an actin filament: a molecular view on the regulation of human formins. Biochim. Biophys. Acta 1803, 152-163. doi: 10.1016/j.bbamcr.2010.01.014

Shin, J. T., Priest, J. R., Ovcharenko, I., Ronco, A., Moore, R. K., Burns, C. G., et al. (2005). Human-zebrafish non-coding conserved elements act in vivo to regulate transcription. Nucleic Acids Res. 33, 5437-5445. doi: 10.1093/nar/gki853

Stainier, D. Y. (2001). Zebrafish genetics and vertebrate heart formation. Nat. Rev. Genet. 2, 39-48. doi: 10.1038/35047564

Teraoka, H., Dong, W., Ogawa, S., Tsukiyama, S., Okuhara, Y., Niiyama, M., et al. (2002). 2,3,7,8-Tetrachlorodibenzo-p-dioxin toxicity in the zebrafish embryo: altered regional blood flow and impaired lower jaw development. Toxicol. Sci. 65, 192-199. doi: 10.1093/toxsci/65.2.192

Thurston, S. F., Kulacz, W. A., Shaikh, S., Lee, J. M., and Copeland, J. W. (2012). The ability to induce microtubule acetylation is a general feature of formin proteins. PLoS ONE 7:e48041. doi: 10.1371/journal.pone.0048041

Tominaga, T., Meng, W., Togashi, K., Urano, H., Alberts, A. S., and Tominaga, M. (2002). The Rho GTPase effector protein, mDia, inhibits the DNA binding ability of the transcription factor Pax6 and changes the pattern of neurite extension in cerebellar granule cells through its binding to Pax6. J. Biol. Chem. 277, 47686-47691. doi: 10.1074/jbc.M207539200

Tran, A. D., Marmo, T. P., Salam, A. A., Che, S., Finkelstein, E., Kabarriti, R., et al. (2007). HDAC6 deacetylation of tubulin modulates dynamics of cellular adhesions. J. Cell Sci. 120(Pt 8), 1469-1479. doi: 10.1242/jcs.03431

Watanabe, N., Kato, T., Fujita, A., Ishizaki, T., and Narumiya, S. (1999). Cooperation between $\mathrm{mDial}$ and ROCK in Rho-induced actin reorganization. Nat. Cell Biol. 1, 136-143. doi: 10.1038/11056

Watanabe, N., Madaule, P., Reid, T., Ishizaki, T., Watanabe, G., Kakizuka, A., et al. (1997). p140mDia, a mammalian homolog of Drosophila diaphanous, is a target protein for Rho small GTPase and is a ligand for profilin. EMBO J. 16, 3044-3056. doi: 10.1093/emboj/16.11.3044

Watanabe, S., De Zan, T., Ishizaki, T., Yasuda, S., Kamijo, H., Yamada, D., et al. (2013). Loss of a Rho-regulated actin nucleator, mDia2, impairs cytokinesis during mouse fetal erythropoiesis. Cell Rep. 5, 926-932. doi: 10.1016/j.celrep.2013.10.021

Whipple, R. A., Cheung, A. M., and Martin, S. S. (2007). Detyrosinated microtubule protrusions in suspended mammary epithelial cells promote reattachment. Exp. Cell Res. 313, 1326-1336. doi: 10.1016/j.yexcr.2007.02.001

Wynshaw-Boris, A., Ryan, G., Deng, C. X., Chan, D. C., Jackson-Grusby, L., Larson, D., et al. (1997). The role of a single formin isoform in the limb and renal phenotypes of limb deformity. Mol. Med. 3, 372-384.

Wyse, M. M., Goicoechea, S., Garcia-Mata, R., Nestor-Kalinoski, A. L., and Eisenmann, K. M. (2017). mDia2 and CXCL12/CXCR4 chemokine signaling intersect to drive tumor cell amoeboid morphological transitions. Biochem. Biophys. Res. Commun. 484, 255-261. doi: 10.1016/j.bbrc.2017.01.087

Wyse, M. M., Lei, J., Nestor-Kalinoski, A. L., and Eisenmann, K. M. (2012). Dia-interacting protein (DIP) imposes migratory plasticity in mDia2dependent tumor cells in three-dimensional matrices. PLOS ONE 7:e45085. doi: 10.1371/journal.pone.0045085

Yamana, N., Arakawa, Y., Nishino, T., Kurokawa, K., Tanji, M., Itoh, R. E., et al. (2006). The Rho-mDial pathway regulates cell polarity and focal adhesion turnover in migrating cells through mobilizing Apc and c-Src. Mol. Cell. Biol. 26, 6844-6858. doi: 10.1128/MCB.00283-06 
Yang, C., Czech, L., Gerboth, S., Kojima, S.-,i., Scita, G., and Svitkina, T. (2007). Novel Roles of formin mDia2 in lamellipodia and filopodia formation in motile cells. PLoS Biol. 5:e317. doi: 10.1371/journal.pbio. 0050317

Zhang, C., Willett, C., and Fremgen, T. (2003). Zebrafish: an animal model for toxicological studies. Curr. Protoc. Toxicol. 17, 1.7.1-1.7.18. doi: 10.1002/0471140856.tx0107s17

Ziske, M. A., Pettee, K. M., Khaing, M., Rubinic, K., and Eisenmann, K. M. (2016). SMIFH2-mediated mDia formin functional inhibition potentiates chemotherapeutic targeting of human ovarian cancer spheroids. Biochem. Biophys. Res. Commun. 472, 33-39. doi: 10.1016/j.bbrc.2016.02.049
Conflict of Interest Statement: The authors declare that the research was conducted in the absence of any commercial or financial relationships that could be construed as a potential conflict of interest.

Copyright (c) 2018 LeCorgne, Tudosie, Lavik, Su, Becker, Moore, Walia, Wisner, Koehler, Alberts, Williams and Eisenmann. This is an open-access article distributed under the terms of the Creative Commons Attribution License (CC BY). The use, distribution or reproduction in other forums is permitted, provided the original author(s) and the copyright owner are credited and that the original publication in this journal is cited, in accordance with accepted academic practice. No use, distribution or reproduction is permitted which does not comply with these terms. 